\title{
Tecnologías sostenibles y su uso en la producción de papa en la región altoandina
}

\author{
J. Caycho-Ronco ${ }^{1}$, A. Arias-Mesia ${ }^{2}$, A. Oswald ${ }^{3}$, R. Esprella-Elias ${ }^{4}$, A. Rivera ${ }^{5}$, F. \\ Yumisaca $^{6}$, J. Andrade-Piedra ${ }^{7}$
}

\section{RESUMEN}

La región de los Andes Centrales de Perú, Ecuador y Bolivia tiene una riqueza única de sistemas de producción de papa. Por miles de años los agricultores de zonas alto-andinas localizadas entre los 3500 y $4200 \mathrm{~m}$ sobre el nivel del mar, han desarrollado tecnologías tradicionales adaptadas a condiciones climáticas severas. Con la finalidad de crear un inventario de técnicas conocidas por los agricultores, se realizó un diagnóstico sobre los sistemas de producción sostenible de papa y se evaluó la situación de la agricultura orgánica en Perú, Ecuador y Bolivia en los años 2006/07. El estudio regional mostró que existen 58 tecnologías tradicionales e innovadoras para todos los pasos comprendidos en la producción de papa (tratamiento de semilla, preparación del suelo, manejo del cultivo, sanidad vegetal, almacenamiento y procesamiento). Estas tecnologías son conservadas principalmente por pequeños agricultores de subsistencia, quienes carecen de recursos económicos o no tienen acceso a mercados en donde pueden adquirir insumos externos. Los agricultores fueron conscientes de los daños a la salud ocasionados por los pesticidas y de la buena calidad organoléptica de la papa producida orgánicamente. Las Organizaciones No Gubernamentales (ONGs) vinculadas a la agricultura mostraron un interés creciente en la producción orgánica ejecutando trabajos que involucran en algunos casos la comercialización de productos orgánicos. Las empresas comercializadoras han comenzado a definir líneas de comercialización exclusivas de insumos orgánicos y se han creado sistemas de fiscalización y programas legales de producción orgánica llevados a cabo por el Estado. Estos resultados permiten definir los lineamientos para desarrollar una estrategia y los conceptos necesarios para vincular a los agricultores de la región Andina a un nicho de mercado específico, considerando la sostenibilidad en sus sistemas de producción.

Palabras clave: Sistemas agrícolas, agricultura orgánica, fertilidad del suelo, control de plagas, certificación.

\section{The use of sustainable technologies in potato production in the Andes}

\begin{abstract}
The Andean regions of Peru, Ecuador and Bolivia harbor a unique potato cropping system richness. For thousands of years, farmers living in the high Andes at 3,500 to 4,200 m a.s.l. have developed technologies adapted to the difficult agro-ecological conditions they face. With the twofold objective of producing an inventory of traditional technologies and evaluating the current development options of organic agricultural systems, surveys were conducted among farmers of the Andean region and stakeholders of the organic agricultural sector in Peru, Bolivia and Ecuador in 2006/07. Based on the

\footnotetext{
${ }^{1}$ Investigador. Centro Internacional de la Papa, Lima, Perú. j.caycho@cgiar.org

${ }^{2}$ Estudiante. Universidad Nacional Agraria La Molina, Lima, Perú. adri4na_a@yahoo.com

${ }^{3}$ Experto Asociado. Centro Internacional de la Papa, Lima, Perú. a.oswald@cgiar.org

${ }^{4}$ Investigador. Promoción e Investigación de Productos Andinos, Cochabamba, Bolivia. r.esprella@proinpalp.org

${ }^{5}$ Estudiante. Escuela Superior Politécnica de Chimborazo, Ecuador. marier125@yahoo.com

${ }^{6}$ Investigador. Instituto Nacional Autónomo de Investigaciones Agropecuarias, Ecuador. fyumisacaj@yahoo.com

${ }^{7}$ Coordinador Regional. Centro Internacional de la Papa, Quito, Ecuador. j.andrade@cgiar.org
} 
information thus gathered, an updated diagnosis of the sector was prepared. The survey recorded 58 traditional and innovative technologies, which comprised the entire production cycle of potato from soil tillage to planting to harvest and storage. These technologies have been preserved mainly by subsistence farming smallholders with little resources and/or limited access to infrastructure and markets. These farmers were also aware of the risk posed by the negative effects of pesticides and of the better organoleptic quality of organically produced potatoes. NGOs working in agricultural development showed a keen interest in the development of the organic sector, some of them being already active in the marketing of organic products. Suppliers of farm inputs and other private enterprises have started to develop organic brand products and certification standards recognized by governmental and international bodies. Hence, the presence of several actors interested in the organic sector and in sustainable agriculture shall facilitate the development of a strategy to link farmers to market niches for products coming from sustainable agriculture production systems.

Key words: agricultural systems, organic agriculture, soil fertility, pest control, certification.

\section{Introducción}

Los Andes Centrales de Perú y Bolivia son el centro de origen de la papa (Solanum spp.) y poseen una amplia diversidad, registrándose más de 3800 variedades diferentes de papas nativas (CIP, 2007). A pesar de la elevada industrialización de la agricultura, la gran mayoría de agricultores en los Andes son pequeños productores con sistemas de producción de subsistencia y comercialización a pequeña escala. Los agricultores de campos localizados entre los 3500 y $4200 \mathrm{~m}$ sobre el nivel del mar han desarrollado métodos de producción de papa con una reducida mecanización del suelo y técnicas de fertilización y fitosanitarias que involucran un uso restringido de fertilizantes químicos y plaguicidas (Altieri, 1996). Dichos sistemas agrícolas tradicionales han sido conservados por generaciones y están adaptados a las condiciones ecológicas y sociales de la región Andina. Sin embargo, existen también pequeños agricultores con niveles de producción comercial y con acceso al mercado, que usan elevadas cantidades de agro-químicos no sólo para incrementar su producción, sino también para satisfacer la demanda del mercado en volumen y calidad (color, tamaño, etc.).

El Centro Internacional de la Papa (CIP) a través del proyecto Papa Andina ${ }^{8}$ está desarrollando una estrategia que vincula pequeños agricultores (de Perú, Ecuador y Bolivia) al mercado considerando como requisito principal la sostenibilidad de sus sistemas de producción (sistemas de agricultura sostenible con enfoque al mercado: SAS-M) (Devaux et al., 2007). El manejo sostenible en este sentido es un concepto dinámico orientado a la necesidad del agricultor de producir para su subsistencia y para el mercado respondiendo a la demandas de calidad, cantidad y continuidad. Los pilares de SAS son el uso racional de los recursos naturales, emplear insumos, productos y tecnologías que no perjudiquen al medioambiente y a la salud de los agricultores y que contribuyan a una producción agrícola durable y económicamente adecuada. El concepto se dirige a toda la diversidad de pequeños productores de papa, por ejemplo a los pequeños productores que usan pocos insumos para prevenir un mal manejo de recursos naturales y para mejorar su productividad usando tecnologías más apropiadas o a los productores comerciales para reducir la cantidad y toxicidad de los agro-químicos que aplican en su campos, y mejorar sus conocimientos de alternativas efectivas.

\footnotetext{
${ }^{8}$ Papa Andina es una iniciativa regional coordinada por el CIP que trabaja con organizaciones de investigación y desarrollo del sector papa en Bolivia, Ecuador y Perú. Su principal objetivo es vincular la investigación al desarrollo para mejorar la seguridad alimentaria y contribuir a la reducción de la pobreza (http://www.papandina.org).
} 
El objetivo de esta propuesta es desarrollar nichos de mercado para productos de papas nativas producidas bajo un manejo sostenible y más saludable para el medio ambiente y el agricultor. En este sentido la gran diversidad de variedades de papas nativas y el conocimiento de tecnologías tradicionales constituyen ventajas comparativas para el acceso a nuevas oportunidades de mercado.

En este contexto se llevó a cabo un estudio sobre tecnologías tradicionales y innovadoras que puedan aportar a sistemas sostenibles de producción de papa y un diagnóstico sobre la situación de la agricultura orgánica en Perú, Ecuador y Bolivia durante los años 2006-07. Se creó un inventario de tecnologías -conocidas o aplicadas por los agricultores- potencialmente adaptables a una producción orgánica y se analizó el rol de los partícipes (actores) potenciales en los SAS-M (agricultores, ONGs, empresas certificadoras y comercializadoras de insumos). Este inventario de técnicas será el primer paso para evaluar y validar tecnologías alternativas (sostenibles o certificadas orgánicamente) de sistemas eficientes de producción de papa que otorguen un valor agregado y permitan a su vez vincular a los agricultores al mercado. Así mismo, servirá para documentar y conservar el conocimiento de las tecnologías usadas tradicionalmente por los agricultores, previniendo su pérdida progresiva ante la introducción y uso de tecnologías dependientes de agro-químicos y el cambio permanente en la producción agraria.

\section{Materiales y métodos}

Se realizó un diagnóstico sobre técnicas tradicionales e innovadoras para una producción de papa sin agro-químicos. Se denominó 'tecnologías tradicionales' a aquellas derivadas de conocimientos comunes, ancestrales, y 'tecnologías innovadoras' a aquellas que fueron enseñadas por técnicos o extensionistas.
Este diagnóstico se llevó a cabo por tres equipos de trabajo en Perú (CIP y Universidad Nacional Agraria La Molina - UNALM), Ecuador (CIP, Instituto Nacional Autónomo de Investigaciones Agropecuarias - INIAP y Escuela Superior Politécnica del Chimborazo - ESPOCH) y Bolivia (Fundación Promoción e Investigación de Productos Andinos - PROINPA) durante los años 2006/07. Se utilizaron encuestas similares, adaptadas a las condiciones locales de cada país. Se entrevistaron a varios actores de los SAS-M y aquellos vinculados a la agricultura orgánica: agricultores, ONGs, empresas certificadoras de producción orgánica, empresas comercializadoras de insumos para la agricultura e instituciones públicas y privadas en los tres países (Tabla 1).

Con la finalidad de recopilar la mayor cantidad de técnicas sostenibles, se seleccionaron comunidades de agricultores de acuerdo a la información proporcionada por ONGs u otras instituciones y otras comunidades ubicadas en zonas remotas y con un escaso acceso a insumos químicos para la producción de papa (Anexo 1). Los agricultores de las comunidades elegidas fueron entrevistados de manera individual 0 grupal usando una encuesta de 20 preguntas parcialmente estructurada (con preguntas abiertas) y diseñada para solicitar información sobre sistemas de cultivo, tecnologías tradicionales de producción conocidas o en uso y su apreciación sobre este tipo de tecnologías.

Las empresas certificadoras de producción orgánica instaladas $o$ que se encontraban solicitando el registro ante el Servicio Nacional de Sanidad Agraria - SENASA (Perú), el Ministerio de Agricultura, Ganadería, Acuacultura y Pesca - MAGAP (Ecuador) o el Servicio Nacional de Sanidad Agropecuaria e Inocuidad Alimentaria - SENASAG (Bolivia) fueron entrevistadas con una encuesta de 19 preguntas solicitando información relacionada a normas, requisitos, marco legal, experiencia en certificación orgánica de papa y su potencial mercado de destino (Anexo 2). 
Tabla 1. Número de actores entrevistados partícipes o involucrados en los sistemas de producción sostenibles y agricultura orgánica

\begin{tabular}{lcccc}
\hline País & $\begin{array}{c}\text { Comunidades de agricultores } \\
\text { (agricultores entrevistados) }\end{array}$ & ONGs & $\begin{array}{c}\text { Empresas } \\
\text { certificadoras }\end{array}$ & $\begin{array}{c}\text { Empresas } \\
\text { comercializadoras }\end{array}$ \\
\hline Perú & $33(287)$ & 22 & 6 & 9 \\
Ecuador & $5(111)$ & 6 & 5 & 5 \\
Bolivia & $7(52)$ & 7 & 3 & 1 \\
Total & $45(450)$ & 35 & 14 & 15 \\
\hline
\end{tabular}

Las empresas comercializadoras de insumos para la agricultura registradas ante el ente regulador de cada país en estudio y que indicaron comercializar insumos para la producción orgánica fueron entrevistadas con una encuesta de 18 preguntas que solicitaba información referente a comercialización de insumos para la producción orgánica (mercado, ventas, costos) (Anexo 2).

Una fuente de información adicional fue una colección de tecnologías de producción de cultivos andinos compilada por el Proyecto Andino de Tecnologías Campesinas -PRATECllevada a cabo en 122 comunidades de ocho regiones del Perú durante un período de aproximadamente 25 años (1987-2002). De igual manera, se usó la información recolectada por la División de Manejo Integrado de Cultivos del CIP, la cual sirvió para validar el uso potencial de las tecnologías indicadas por los agricultores. Complementariamente, los investigadores del Programa de Papa del Instituto Nacional de Innovación Agraria (INIA), INIAP y PROINPA fueron consultados para describir tecnologías locales específicas y ayudar a interpretar terminologías en Aymara o Quechua.

\section{Resultados y discusión}

\section{Comunidades de agricultores}

Se encuestaron agricultores de 45 comunidades en total en los tres países. Las regiones en las que realizaron las encuestas fueron la provincia de Chimborazo en Ecuador; departamento de La Paz en Bolivia; y departamentos de Cajamarca, La Libertad, Ancash, Lima, Junín, Huancavelica, Ayacucho, Apurimac, Cusco y Puno en Perú (Tabla 1 y Anexo 1).

Para el 88\% de agricultores en Perú y todos los agricultores de Ecuador y Bolivia el cultivo de papa fue el más importante. Otros cultivos de importancia fueron la cebada (Hordeum vulgare L.), quinua (Chenopodium quinoa W.), haba (Vicia faba L.) y maíz (Zea mays L.) sembrados usualmente en rotación con papa. El $87 \%$ de agricultores en Ecuador indicó usar insecticidas y/o fertilizantes químicos en el cultivo de papa mientras en Perú y Bolivia la cifra fue más baja con $71 \%$ y $31 \%$ respectivamente. Esto refleja parcialmente la finalidad de la producción de papa con una producción principalmente comercial en Ecuador, una mezcla de producción comercial y de subsistencia en Perú y una producción principalmente de subsistencia en el Altiplano de Bolivia.

Se registraron un total de 58 técnicas diferentes en los tres países, 54 técnicas en Perú con el estudio más extenso y la información adicional de PRATEC, 19 técnicas en Ecuador, de las cuales 2 fueron propias a este país y 19 en Bolivia con 2 técnicas diferentes a las mencionadas en Perú y Ecuador (Tabla 2). 
Tabla 2. Técnicas sostenibles mencionadas por los agricultores e instituciones entrevistadas en los tres países en estudio

\begin{tabular}{|c|c|c|c|c|}
\hline & Técnicas mencionadas & Perú & Ecuador & Bolivia \\
\hline \multirow[t]{8}{*}{ Preparación del suelo } & Barbecho & $\mathrm{X}$ & $\mathrm{X}$ & $\mathrm{X}$ \\
\hline & Tipka o Chiwa & $\mathrm{X}$ & & \\
\hline & Chacma & $\mathrm{X}$ & & \\
\hline & Wachu rozado & & $\mathrm{X}$ & \\
\hline & Andenes o terrazas & $\mathrm{X}$ & $\mathrm{X}$ & $\mathrm{X}$ \\
\hline & Waru waru & $\mathrm{X}$ & & $\mathrm{X}$ \\
\hline & Clasificación de suelos & $\mathrm{X}$ & & \\
\hline & Recuperación de suelos erosionados & $\mathrm{X}$ & & \\
\hline \multirow[t]{9}{*}{ Fertilización } & Aplicación de estiércol & $\mathrm{X}$ & $\mathrm{X}$ & $\mathrm{X}$ \\
\hline & Majadeo & $\mathrm{X}$ & $\mathrm{X}$ & \\
\hline & Quema de rastrojos y malezas o Japucha & $\mathrm{X}$ & & $\mathrm{X}$ \\
\hline & Jiracha & & & $\mathrm{X}$ \\
\hline & Aplicación de biol & & $\mathrm{X}$ & $\mathrm{X}$ \\
\hline & Aplicación de humus de lombriz & $\mathrm{X}$ & $\mathrm{X}$ & $\mathrm{X}$ \\
\hline & Aplicación de gallinaza & $\mathrm{X}$ & $\mathrm{X}$ & \\
\hline & Aplicación de purín de ganado & $\mathrm{X}$ & $\mathrm{X}$ & \\
\hline & Incorporación de abonos verdes & & $\mathrm{X}$ & \\
\hline \multirow{5}{*}{$\begin{array}{l}\text { Manejo de riesgos } \\
\text { climáticos }\end{array}$} & Siembra en mezcla, chaqro o chagro. & $\mathrm{X}$ & & $\mathrm{X}$ \\
\hline & Asociación de cultivos* & $\mathrm{X}$ & & \\
\hline & Ch'eje & $\mathrm{X}$ & & \\
\hline & Qocha & $\mathrm{X}$ & & \\
\hline & Pirca & $\mathrm{X}$ & & \\
\hline \multirow{14}{*}{$\begin{array}{l}\text { Control de plagas } \\
\text { en campo }\end{array}$} & Rotación de cultivos & $\mathrm{X}$ & $\mathrm{X}$ & $\mathrm{X}$ \\
\hline & Rotación sectorial & $\mathrm{X}$ & & $\mathrm{X}$ \\
\hline & $\begin{array}{l}\text { Aplicación de extractos repelentes o } \\
\text { biocidas }\end{array}$ & $\mathrm{X}$ & $\mathrm{X}$ & $\mathrm{X}$ \\
\hline & Barreras vivas & $\mathrm{X}$ & $\mathrm{X}$ & $\mathrm{X}$ \\
\hline & Aplicación de ceniza* & $\mathrm{X}$ & $\mathrm{X}$ & \\
\hline & Aplicación de cal* & $\mathrm{X}$ & $\mathrm{X}$ & $\mathrm{X}$ \\
\hline & Quema de plantas repelentes y azufre & $\mathrm{X}$ & & \\
\hline & Aporque alto & $\mathrm{X}$ & & \\
\hline & Recolección manual de insectos & $\mathrm{X}$ & & \\
\hline & Cosecha oportuna & $\mathrm{X}$ & & \\
\hline & Construcción de zanjas & $\mathrm{X}$ & & \\
\hline & Uso de trampas amarillas & $\mathrm{X}$ & & \\
\hline & Uso de barreras plásticas & $\mathrm{X}$ & & \\
\hline & Uso de trampas de caída & $\mathrm{X}$ & & \\
\hline \multirow[t]{3}{*}{ Control de enfermedades } & Aplicación de extractos de plantas biocidas & s $\mathrm{X}$ & & \\
\hline & Aplicación de bioles y/o caldo bordalés & $\mathrm{X}$ & $\mathrm{X}$ & \\
\hline & Aplicación de orina fermentada* & $\mathrm{X}$ & & \\
\hline \multirow{8}{*}{$\begin{array}{l}\text { Control de plagas } \\
\text { en almacén }\end{array}$} & Uso de plantas repelentes* & $\mathrm{X}$ & & $\mathrm{X}$ \\
\hline & Selección y sellado del silo & & & $\mathrm{X}$ \\
\hline & Aplicación de Beauveria sp. & $\mathrm{X}$ & & \\
\hline & Aplicación de Baculovirus & $\mathrm{X}$ & & $\mathrm{X}$ \\
\hline & Uso de feromonas & $\mathrm{X}$ & & \\
\hline & Aplicación de agua jabonosa & $\mathrm{X}$ & & \\
\hline & Aplicación de hollín & $\mathrm{X}$ & & \\
\hline & Humeo de ají & $\mathrm{X}$ & & \\
\hline \multirow[t]{3}{*}{ Tratamiento de semilla } & Selección de semilla & $\mathrm{X}$ & $\mathrm{X}$ & $\mathrm{X}$ \\
\hline & Verdeamiento & $\mathrm{X}$ & $\mathrm{X}$ & \\
\hline & Jiri o Jamallachi & $\mathrm{X}$ & & \\
\hline
\end{tabular}




\begin{tabular}{lllll}
\hline \multirow{3}{*}{ Almacenamiento } & K'ayro o Phyna & $\mathrm{X}$ & $\mathrm{X}$ & $\mathrm{X}$ \\
& Tarimas de madera & $\mathrm{X}$ & & \\
& Estructuras de adobe o piedra & $\mathrm{X}$ & \\
\hline Procesamiento** & Elaboración de chuño blanco & $\mathrm{X}$ & & \\
& Elaboración de chuño negro & $\mathrm{X}$ & & \\
& Elaboración de papa seca & $\mathrm{X}$ & & \\
& Elaboración de harina & $\mathrm{X}$ & \\
\hline
\end{tabular}

* Técnicas mencionadas en más de una etapa de la producción de papa

** Registradas por PRATEC

Las técnicas recolectadas representan toda la cadena productiva en el cultivo de papa, desde preparación del suelo hasta procesamiento (Figura 1). Las técnicas pueden estar distribuidas uniformemente en los tres países o ser conocidas o aplicadas solamente en comunidades específicas en una región. La mayoría de técnicas registradas fueron tradicionales $(76 \%)$ y se reportaron también técnicas 'innovadoras' (24\%) promovidas por ONGs o instituciones públicas o privadas de investigación agraria (aplicación de Beauveria sp. y Baculovirus, uso de feromonas, etc.).

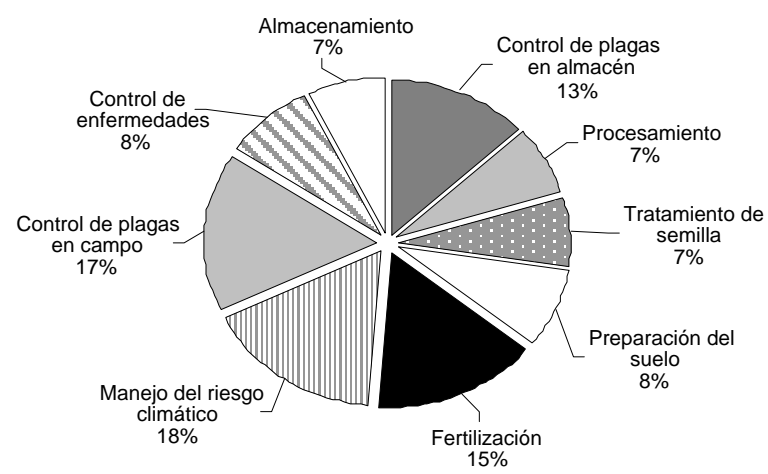

Figura 1. Distribución de las tecnologías de producción de papa en los Andes Centrales clasificadas de acuerdo a su uso.

Gran parte de las tecnologías pertenecen a dos áreas cruciales de la producción de papa: la provisión de nutrientes y fertilidad del suelo y el control de plagas en campo y almacén. En los siguientes capítulos se analiza con más detalle las diferentes tecnologías antes de reportar el rol del sector privado y de ONGs involucradas en una producción agraria sostenible.

\section{Preparación del suelo}

Se mencionaron diferentes técnicas tradicionales de preparación del suelo utilizadas por los agricultores en el cultivo de papa (Tabla 2). El tipo de labranza más usado y distribuido en casi todas las regiones es el barbecho mientras la chacma y tipka son conocidas en zonas específicas de Perú.

El barbecho o qholli es una labranza que consiste en la remoción total del suelo durante las últimas lluvias. Con el comienzo de la nueva estación de lluvias se hace surcos de textura fina para proporcionar buenas condiciones de crecimiento para la papa. En la chacma se voltean terrones del suelo entre los cuales se siembra el tubérculo de papa formándose surcos de menor textura y con un espacio reducido para el desarrollo de los tubérculos. En la tipka (conocida también como chiwa, imicha, crudo o tipca) se levanta un terrón de suelo, se siembra el tubérculo y se cubre con el mismo suelo. Después de algunas semanas el agricultor levanta el suelo de ambos lados de las papas sembradas para formar surcos. Esta técnica es menos intensiva que las dos anteriores, sin embargo, las plantas necesitan más tiempo para emerger. El barbecho permite el mantenimiento de la humedad en el suelo y la exposición de las plagas del suelo; mientras que la chacma y la tipka son tipos de labranza mínima adaptadas a condiciones de topografía accidentada y que han sido desarrolladas para evitar la erosión (de agua y viento) y cultivar sobre pendientes severas. La tipka es usualmente practicada para producir variedades nativas en las zonas más altas de los Andes (superiores a los $3800 \mathrm{~m}$ sobre el nivel del mar) y tiene un rendimiento menor y tubérculos más pequeños en comparación al chacmeo o barbecho. La herramienta principal para la 
roturación es la chakitaklla (arado de pie o tirapie utilizado para voltear o remover el terreno) (Borliaud et al., 1988; Cóndor, 1994; Tapia y Fries, 2007).

En Ecuador, se registró un tipo de labranza llamada wachu rozado que posee características similares a la chacma (Pérez, 1996; Pumisacho y Sherwood, 2002).

Otra técnica para la conservación del suelo es la construcción de andenes. Los andenes son terrazas 0 construcciones de plataformas continuas escalonadas en las laderas de los cerros y superficies inclinadas de las quebradas. De este modo se logra el aprovechamiento óptimo del terreno y del agua, se reduce el riesgo de las bajas temperaturas (heladas) y se evita la erosión del suelo (Blossiers et al., 2000). Altieri (1996) indica que la superficie de andenes en el Perú es de aproximadamente 500000 hectáreas. En la actualidad no se construyen nuevos andenes; sin embargo, los andenes abandonados o derrumbados son rehabilitados en algunas regiones.

En el Altiplano se conoce la construcción de waru-warus o sukakollos que consisten en plataformas de suelo o camellones levantados artificialmente y rodeados de canales o surcos llenos de agua para mejorar el drenaje y disminuir el efecto de factores abióticos adversos (Borliaud et al., 1988; Erickson, 1984). El agua de los surcos retiene la energía solar durante el día y la irradia durante la noche, disminuyendo así el impacto de las heladas sobre los cultivos (Erickson y Chandler, 1989). La retención de agua en los canales ayuda a mantener la humedad en las raíces de los cultivos durante las sequías y la acumulación de residuos vegetales y animales en descomposición crea una capa de suelo rica en nutrientes que mejora la fertilidad de los camellones (Altieri, 1996).

Los agricultores tienen además diferentes sistemas de clasificación de suelos de acuerdo al color, profundidad y textura y técnicas de recuperación de suelos erosionados, como por ejemplo la rotura de la capa superficial del suelo erosionado y aplicación de estiércol antes de la siembra durante varios años.

\section{Fertilización}

La aplicación de estiércol durante la siembra fue la técnica mas reportada por los agricultores para la fertilización del cultivo y del suelo (Tabla 2). En Perú y Ecuador se registró una técnica tradicional conocida como majadeo, ishpachi o talanqueras que consiste en estabular al ganado (ovino, camélido o caprino) durante las noches en un corral "móvil” de construcción simple. El objetivo es permitir la mezcla de las deyecciones acumuladas con el suelo del campo mediante el pisoteo constante del ganado y el ingreso de nutrientes de terrenos cercanos al campo que será sembrado en la siguiente campaña. La remoción del terreno al término del majadeo con la finalidad de incorporar el estiércol al suelo se conoce como tzaumar en Ecuador. Los agricultores mencionaron que antiguamente sólo abonaban con majada de animales y utilizaban ceniza del fogón que también ayudaba al control de plagas y enfermedades.

En Perú y Bolivia se usa una técnica llamada japucha que consiste en apilar rastrojos $\mathrm{y}$ malezas en distintos lugares de la parcela durante el desterronamiento o k'upa para luego quemar estos montones e incorporar posteriormente la ceniza al suelo. Es una manera rápida de deshacerse de grandes volúmenes de restos de cosecha y malezas. En Bolivia, el $87 \%$ de agricultores indicó fertilizar el suelo con jiracha (mezcla de estiércol ovino o aviar fermentado y agua).

La utilización de abonos orgánicos fue otra técnica de fertilización destacando entre ellos la aplicación de bioles obtenidos a partir de la descomposición anaeróbica de insumos orgánicos (estiércol, rastrojos, azúcar y otros ingredientes) propios de cada zona. Otras fuentes de fertilización orgánica fueron la aplicación de humus de lombriz, gallinaza (mezcla de estiércol de gallina y cascarilla de arroz o viruta), purín de ganado e incorporación de abonos verdes.

La mayoría de los agricultores en los tres países reportó que papas producidas sólo con 
fertilizantes orgánicos tienen un mejor sabor, un contenido de materia seca más alta, menor tiempo de cocción, una mejor capacidad de almacenamiento y generalmente una mejor calidad. (Figura 2). También fueron conscientes que el uso de agro-químicos puede contaminar el suelo y los productos agrícolas, poner en peligro su salud y tener otras consecuencias negativas, como por ejemplo una reducida productividad.

Por esta razón en varias comunidades los agricultores reservan parcelas para su producción de subsistencia y las manejan sin uso de agroquímicos mientras mantienen paralelamente su producción comercial manejada de forma convencional para el mercado.

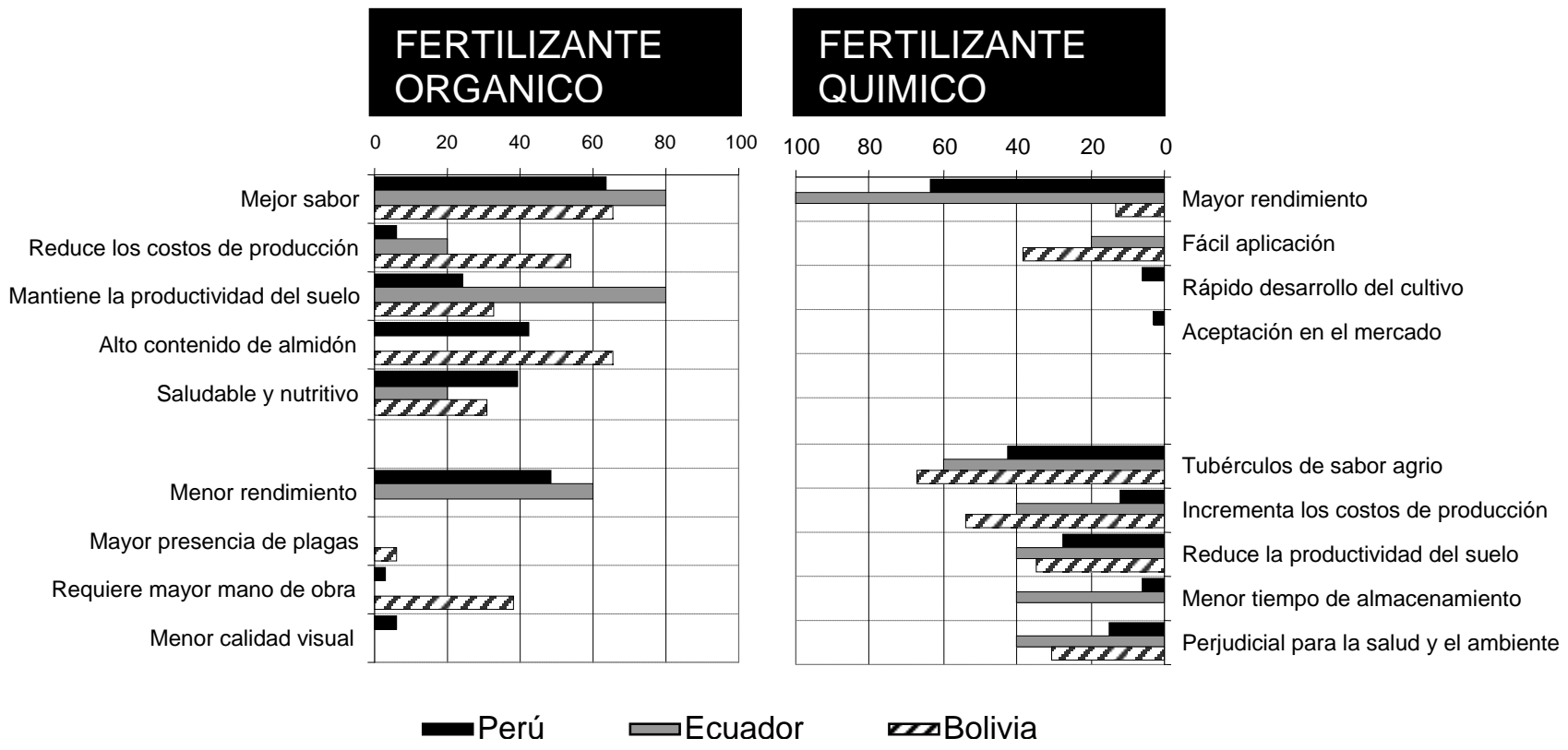

Figura 2. Percepción de los agricultores en la aplicación de fertilizantes en el cultivo de papa en las comunidades de Perú; Ecuador y Bolivia.

\section{Manejo de riesgos climáticos}

La asociación de diferentes cultivos con la papa y la siembra de diferentes variedades de papas nativas en una misma parcela conocida también como siembra en mezcla, chaqro, chalo o chaccro son prácticas comunes en las zonas altoandinas de Perú y Bolivia, y en menor grado en Ecuador. Esta diversidad genética en campo permite disminuir la incidencia de plagas (Ewell et al., 1994) además de mitigar el riesgo de daños por factores abióticos (heladas, sequías) y cumplir un rol importante en la conservación in situ de la diversidad de papas nativas (Brush et al., 1979).
Adicionalmente se registraron otras técnicas que reducen el daño ocasionado por sequías o heladas en el cultivo de la papa. Entre ellas tenemos los ch'ejes o estructuras circulares de piedra que absorben el calor en el día y lo liberan durante la noche evitando cambios bruscos de temperatura; las qochas o depósitos en donde se almacena el agua de las lluvias que es luego utilizada en época de sequía; las pircas o cercos de piedra alrededor de la parcela para proteger los cultivos contra los daños del ganado, el viento o heladas y la siembra de variedades resistentes a heladas (puqya y waña). 


\section{Protección vegetal}

Los agricultores han desarrollado diferentes estrategias de control de plagas y enfermedades. La rotación de cultivos fue la principal técnica mencionada por los agricultores de Perú, Bolivia y Ecuador para el control de plagas en campo especialmente del gorgojo de los Andes (Premnotrypes sp.), una de las plagas más importantes en la región andina (Alcázar y Cisneros, 1999) y para mantener o mejorar la fertilidad del suelo (Figura 3).

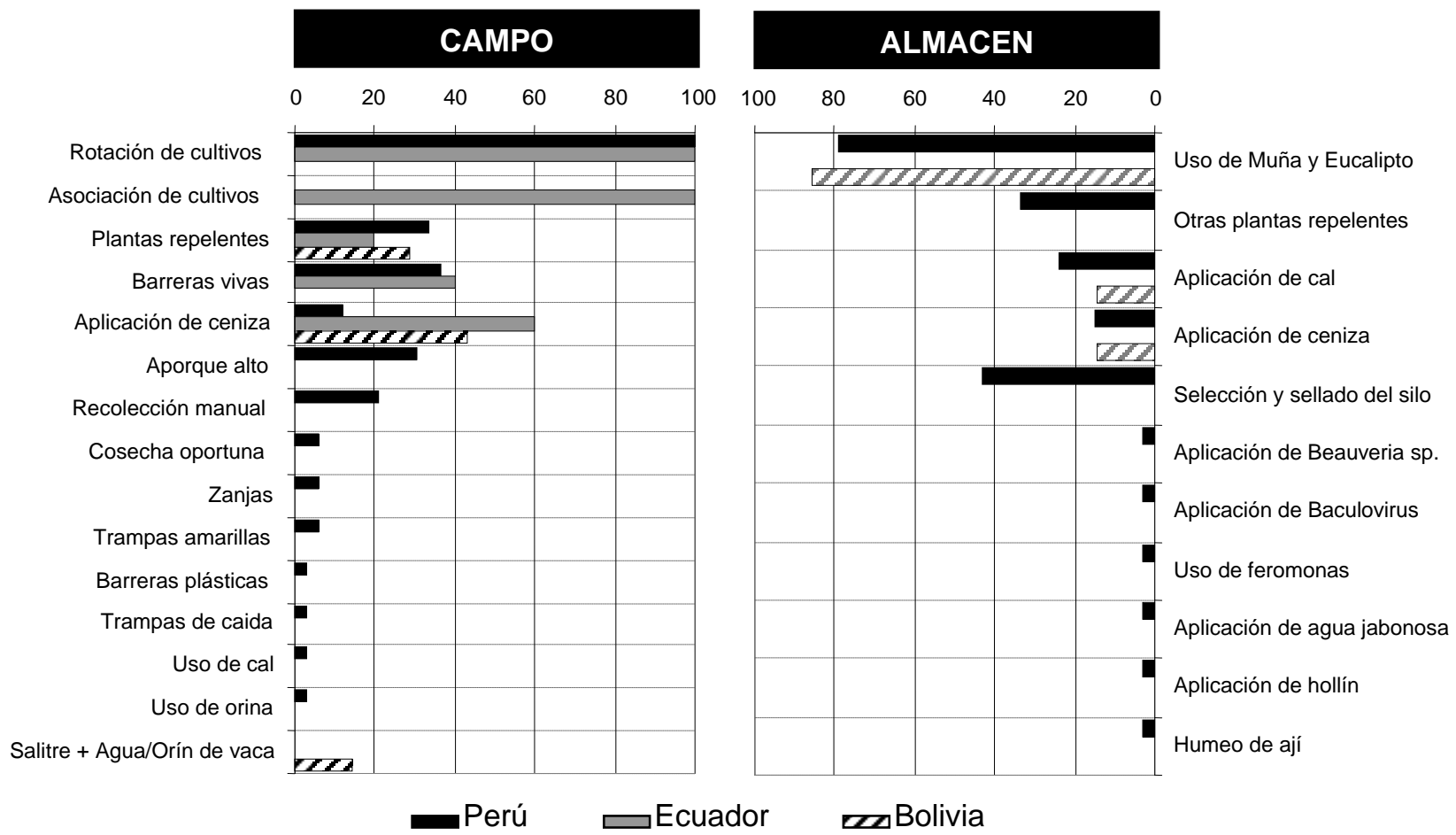

Figura 3. Técnicas referidas a la sanidad vegetal del cultivo de papa mencionadas por los agricultores de Perú; Ecuador y Bolivia.

Los cultivos utilizados en la rotación así como el período de descanso varían de acuerdo a cada región, zona agro-ecológica, disponibilidad de terreno y presión demográfica. El orden de rotación predominante es papa - granos (andinos) - leguminosas o forraje y descanso (210 años). Adicionalmente se reportó una rotación sectorial de cultivos conocida también como barbecho sectorial, turnos, laymes o aynoqas referida a la rotación de terrenos comunales. En este tipo de rotación el terreno comunal es dividido en zonas dedicadas a un solo cultivo y que son rotadas cada año. La participación de la comunidad es colectiva constituyendo una distribución social de los terrenos (tierras de propiedad comunal en usufructo). Los agricultores afirman que las aynoqas se usan por tradición o costumbre prevaleciente y porque resulta en un buen manejo de plagas y del suelo.
La utilización de extractos repelentes o biocidas fue otra técnica tradicional. La preparación del extracto y el tipo de planta varían de acuerdo a cada región (Tabla 3). En el Perú existen más de 350 especies vegetales registradas con potencial biocida (Gomero, 2000) y su uso podría constituir una alternativa en el manejo ecológico de plagas, reduciendo los costos de producción y la utilización de insumos externos.

La siembra de tarhui (Lupinus mutabilis Sweet.) $\mathrm{u}$ oca (Oxalis tuberosa Mol.) alrededor del cultivo de papa a manera de cercos o barreras vivas constituye un método para el control de Premnotrypes sp. y para evitar que el ganado ingrese al campo. A pesar de que esta técnica es ampliamente conocida su uso no es frecuente. 
Tabla 3. Plantas utilizadas por los agricultores para el control de plagas

\begin{tabular}{lll}
\hline \multicolumn{1}{c}{ Nombre local } & \multicolumn{1}{c}{ Nombre científico } & \multicolumn{1}{c}{ Plaga que controla } \\
\hline Ajenjo & Artemisia absinthium L. & Premnotrypes spp. \\
Ajo & Allium sativum L. & Premnotrypes spp.; mosca blanca; áfidos \\
Cola de caballo & Equisetum bogotense HBK. & Trips (Frankliniella spp.) \\
Chamcua, chamca, muña & Minthostachys setosa (Biq.) Epling & No especificada \\
Geranio & Pelargonium spp. & Mosca blanca \\
Maicha & Senecio pseudotites Griseb. & Áfidos Myzus persicae Sulzar y \\
& & Macrosiphum euphorbiae Thomas \\
Ortiga & Urtica spp. & ÁfidosMyzus persicae Sulzar y \\
& & Macrosiphum euphorbiae Thomas \\
Pupusa & Werneria digitata Wedd. & No especificada \\
Rocoto & Capsicum pubescens Ruiz y Pavón & Premnotrypes spp.; Diabrotica spp.; \\
& & Trips (Frankliniella spp.); mosca blanca; \\
Sasahui & áfidos \\
Sauco & Leucheria sp. & Áfidos \\
Supai-ccarco & Sambucus peruviana HBK. & Mosca blanca \\
Tara & Nicotiana glauca Graham & Mosca blanca \\
Tarhui & Caesalpinia spinosa (Molina) Kuntze. & No especificada \\
\hline Fun & Lupinus mutabilis Sweet & Premnotrypes spp. \\
\hline
\end{tabular}

Fuente: Brack, 1999

El espolvoreo de ceniza y/o cal alrededor de la planta es una práctica común para el control de plagas (Premnotrypes sp., Diabrotica sp. y Epicauta sp.) y enfermedades (Phytophthora infestans). En Perú se mencionó la aplicación de ceniza de quinua (Chenopodium quinoa (Willd)), eucalipto (Eucalyptus globulus Labill), tarhui y estiércol seco de vacuno para el control de plagas. En Bolivia la aplicación de ceniza de k’oa (Satureja boliviana Briq.), ñaqa thola (Lepidophylla sp.) o supu thola (Baccharis sp.) antes de la etapa de floración sirve para controlar o prevenir el ataque de Premnotrypes sp. y Epitrix sp. También se usa el humo de la combustión del azufre combinado con una infusión de choquecaylla (Margyricarpus setosus Ruiz y Pavón) y k’oa espolvoreada para el control de plagas en campo. La quema de estos insumos se realiza por separado a un extremo de la parcela, en dirección del viento, para que las plantas queden impregnadas con un sabor picante.

Otras técnicas para control de plagas de campo son: aporque alto que consiste en la remoción del suelo para cubrir el cuello de la planta dificultando el ingreso de insectos y/o patógenos; cosecha oportuna evitando que el tubérculo permanezca en campo reduciendo el daño ocasionado por los gusanos de tierra (Copitarsia sp.); construcción de zanjas para impedir que los adultos de gorgojo ingresen a los campos recién sembrados (Yabar, 1988).

Con relación al control de plagas en almacén, es frecuente el uso de plantas repelentes como muña (Minthostachys spp.) o eucalipto en Perú y k’oa en Bolivia. Las plantas repelentes pueden ser distribuidas a manera de capas entre los tubérculos almacenados; molidas y posteriormente espolvoreadas o quemadas $\mathrm{y}$ aplicadas como ceniza (Canales y Canto, 2000). 
Otras técnicas (para campo o almacén) como el uso de trampas amarillas, trampas de caída y barreras plásticas, aplicación de Beauveria sp. o Baculovirus phthorimaea y el uso de feromonas han sido introducidas o recomendadas por ONGs o instituciones de investigación en comunidades específicas. Sin embargo, su uso es muy limitado.

Se registró un menor número de técnicas para el control de enfermedades en comparación al control de plagas (Figura 1). En Perú, se aplica extractos de plantas locales con acción fungicida y ceniza u orina fermentada sobre el follaje para controlar $P$. infestans u otros patógenos foliares. En Ecuador los agricultores no diferenciaron la acción de plagas y enfermedades sobre sus cultivos, al aplicar un determinado insumo para controlar ambos casos. Por ejemplo, ceniza aplicada sobre las plantas y adicionalmente cal en el suelo puede servir para prevenir enfermedades y controlar plagas e incluso para fertilizar el suelo. Similar caso ocurre con bioles preparados a base de cola de caballo (Equisetum sp.), manzanilla (Matricaria chamomilla L.), leche y melaza. Generalmente los agricultores son conscientes de la importancia de mantener el suelo con vida y que el uso irracional de plaguicidas puede disminuir la productividad de sus tierras.

En Bolivia, no se recolectaron técnicas aplicadas para el control de enfermedades debido a que la incidencia de éstas no es frecuente en la zona del Altiplano.

En varios casos existe evidencia científica sobre la efectividad de estas tecnologías. Por ejemplo, se conoce el efecto de la rotación de cultivos en patógenos de suelo como Verticillium dahliae (Davis et al., 1994) y actualmente la rotación es una tecnología ampliamente recomendada para controlar este tipo de patógenos (Agrios, 1997). Otro ejemplo es el uso de muña (Minthostachys spp.) que en estudios preliminares ha mostrado su potencial como inhibidor de brotación frente a productos sintéticos como CIPC (chlorpropham) (K. Manrique, comunicación personal). Sin embargo, en otros casos es necesario realizar investigaciones para determinar el efecto de las tecnologías aplicadas por agricultores, por ejemplo el uso de ceniza para control de $P$. infestans o el uso de tarwi para control del gorgojo, que según experimentos ejecutados en el Altiplano no tiene efecto repelente (J. Arcos, comunicación personal).

\section{Tratamiento de semilla y almacenamiento}

Los agricultores han desarrollado diferentes técnicas para tratar y almacenar semilla de papa, siendo la más utilizada la selección de semilla basada en el tamaño y calidad sanitaria de los tubérculos y realizada inmediatamente después de la cosecha (Fonseca et al., 2002). En Perú y Ecuador se registró también la técnica de verdeamiento realizada luego de la selección y que consiste en la exposición de los tubérculos a la luz indirecta o difusa (bajo sombra) para estimular la formación de solanina y clorofila favoreciendo una brotación múltiple vigorosa y la reducción en la incidencia de plagas (Murcia y Barreto, 2002). En Perú se mencionó la aplicación del estiércol -conocido como Jiri, Jamallach'i o Ch'ojña Jiri- directamente a la semilla antes de la siembra. El jiri es el estiércol fermentado de forma anaerobia, en consistencia pastosa, color verde oscuro y olor característico ubicado en la capa profunda del corral (Valdez, 1993). Este tipo de estiércol posee propiedades desinfectantes actuando como ovicida y larvicida al ser untado en la semilla y tiene propiedades cicatrizantes, cuando la semilla se utiliza en forma fraccionada o cuando tiene daños mecánicos.

El almacenamiento de la papa juega un rol importante para el agricultor de zonas altoandinas al asegurar la alimentación de su familia, la disponibilidad de la semilla y la mejora de sus ingresos económicos (Fonseca et al., 2002). La papa es tradicionalmente almacenada en k'airos (hoyos en el suelo de 30-50 cm de profundidad cerca de la casa) o phynas (almacenamiento temporal en las parcelas). En Perú se mencionó el uso de tarimas de madera sobre las cuales se colocan las semillas utilizadas también para el control de Premnotrypes spp. ya que las larvas caen a través de las ranuras. También se usan depósitos rectangulares o circulares de adobe o piedra (60-70 cm de altura) construidos fuera o 
dentro de las viviendas en donde se almacenan los tubérculos de papa cubiertos con plantas repelentes locales. Algunas de estas estructuras constan de tres niveles, para almacenamiento de tubérculos $\left(1^{\mathrm{er}}\right.$ nivel), granos $\left(2^{\mathrm{do}}\right.$ nivel $) \mathrm{y}$ herramientas $\left(3^{\mathrm{er}}\right.$ nivel).

\section{Procesamiento}

La alimentación de los agricultores alto-andinos ha sido facilitada por la elaboración de chuño. El chuño blanco o moraya (quechua) o tunta (aymara) se produce a partir de las papas amargas (alto contenido de glicoalcaloides), a través de un proceso de deshidratación natural aprovechando las condiciones climáticas altoandinas. Los tubérculos se someten a congelación nocturna durante las heladas de junio y julio y de día a la fuerte insolación (CIP, 2003). Otra calidad de chuño es el chuño negro, que se elabora a partir de los tubérculos pequeños de las variedades de papa nativa común y de algunas mejoradas. Su proceso requiere de menos cuidado, no es remojado en los ríos, y solamente es expuesto de cinco a diez días -sin ninguna protección - a las heladas nocturnas y a la fuerte radiación solar, obteniendo así el color negro característico (Gianella, 2004). El chuño se puede almacenar por años sin que pierda su calidad culinaria. Sin embargo, en el proceso de conservación la papa pierde su contenido de vitamina $C$, por ser ésta hidrosoluble, pero durante el proceso de elaboración se incrementa notablemente el contenido de calcio y hierro.

La papa seca es un producto que incrementa la durabilidad de la papa. En el proceso se pela, lava y cuece los tubérculos y luego se corta y seca la papa en hornos o en aire libre con el sol. Finalmente se puede moler los trozos haciendo una harina (Gómez y Wong, 1998).

\section{ONGs, empresas certificadoras y empresas de insumos agrícolas}

Se entrevistaron un total de 35 ONGs en los tres países en estudio (Tabla 1 y Anexo 2). El 60\% de ONGs definió el concepto de producción orgánica como el "no uso de agroquímicos”, un 18\% mencionó adicionalmente aspectos de sostenibilidad, conservación de recursos naturales y salud humana como puntos importantes, el 6\% afirmó que la certificación es esencial para considerar a un cultivo como orgánico; mientras que el $11 \%$ no tuvo una idea clara sobre la producción orgánica. Esto indica que el concepto de producción agrícola sostenible u orgánica no es muy claro entre las ONGs, aunque la mayoría de ellas señalaron que existe un creciente interés de las comunidades en este tema por tres principales razones: (1) salud, al considerar a la producción orgánica como un sistema que no atenta al bienestar de los agricultores y consumidores; (2) económica, al obtener productos con valor agregado, ya que según los entrevistados, el mercado consumidor comienza a tener conciencia de los beneficios de los productos orgánicos; y (3) ambiental, al considerar la conservación y menor contaminación del medio ambiente.

Los agrónomos de las ONGs no conocieron técnicas diferentes a las mencionadas por los agricultores, a excepción de la utilización de insumos certificados (elaborados principalmente a partir de Bacillus thuringiensis Berliner, Beauveria sp., Paecilomyces lilacinus (Thom) Samsom, o Allium sativum L.) y permitidos en la producción orgánica y la aplicación de azufre y cobre para el control de enfermedades.

Todas las empresas de certificación orgánica entrevistadas están registradas ante la autoridad competente en materia de producción orgánica en cada país contando con la acreditación para los mercados estadounidense (National Organic Program - United States Department of Agriculture), europeo (European Union Regulation No. 2092/91) y japonés (Japanese Agricultural Standard); a excepción de la Asociación de Organizaciones de Productores Ecológicos de Bolivia (AOPEB) que usa el sistema de certificación participativa. Estas empresas coincidieron en señalar que el agricultor que requiere una certificación orgánica debe informarse previamente sobre el sistema, normas y/o técnicas de producción orgánica; ya que las empresas certificadoras no realizan ningún tipo de asesoría en este rubro, limitándose tan solo a la certificación. Las 
empresas certificadoras han identificado tres segmentos en sus clientes: (1) grupo de agricultores que reconocen los beneficios de la producción orgánica; (2) grupo de agricultores a los cuales se les certifica "por defecto" debido a que no utilizan agro-químicos, citando como ejemplo a los productores alto-andinos de quinua orgánica; y (3) grupos de agricultores que se interesan en producción orgánica sólo por el margen adicional que ésta representa en sus ventas en comparación a la agricultura convencional. Las empresas certificadoras señalan que los agricultores recurren principalmente a sus insumos locales y son pocos los que usan productos comerciales en su producción orgánica. La AOPEB mencionó tres pasos necesarios que los agricultores deben seguir para acceder a una Certificación Participativa dentro del Sistema de Garantía Participativa: (1) formular sus propias normas (adecuadas a las normas del International Federation of Organic Agriculture Movements IFOAM; (2) elaborar instrumentos de control e inspección interna; y (3) recibir la inspección de un organismo de control acreditado para obtener el certificado ecológico. Otra diferencia entre las certificadoras convencionales y la AOPEB es el costo de la certificación. Para pequeños agricultores este costo es muy elevado, siendo necesario que estén organizados (por ejemplo, en comunidades) para obtener una certificación, muchas veces con subsidios de ONGs. La AOPEB ha reducido significativamente el costo de certificación a través de su sistema de control interno y participativo, logrando el acceso a los agricultores andinos. Sólo Bio-Latina en Perú y la AOPEB en Bolivia indicaron estar en proceso de certificación orgánica de papa.

Varias empresas de comercialización de insumos agrícolas señalaron al mercado de la agroexportación como el principal consumidor de insumos para la producción orgánica. Las exigencias establecidas en los Límites Máximos de Residuos para Plaguicidas (LMR) para todos los alimentos destinados al consumo humano o animal en los principales mercados internacionales (Unión Europea, Estados Unidos y Japón) obligan a las empresas agroexportadoras a cumplir tales normas de inocuidad usando insumos de baja toxicidad y cuyo uso está permitido en la producción orgánica. De este modo las ventas de insumos certificados orgánicamente son significativas para los agro-exportadores y esporádicas o eventuales para los agricultores orgánicos. Sin embargo, las empresas comercializadoras entrevistadas ven a la producción orgánica como un mercado potencial, debido al incremento de los volúmenes de exportación de productos orgánicos en los últimos años.

\section{Conclusiones}

Este estudio regional mostró que existen 58 tecnologías tradicionales e innovadoras para todos los pasos comprendidos en la producción de papa (tratamiento de semilla, preparación del suelo, manejo del cultivo, sanidad vegetal, almacenamiento y procesamiento). Muchas de estas tecnologías han co-evolucionado desde que el cultivo de papa llegó a ser el principal alimento de la región miles de años atrás y están adaptadas específicamente a las condiciones agro-ecológicas y climáticas de la región andina. Estas tecnologías son conservadas principalmente por pequeños agricultores de subsistencia, quienes carecen de recursos o no tienen acceso a mercados en donde pueden adquirir insumos externos. Muchas veces los agricultores saben de los riesgos del uso de pesticidas para su salud y para el medioambiente pero son reacios a reducir o suprimir completamente el uso de agroquímicos, ya sea por falta de conocimiento o de alternativas efectivas, especialmente en el caso de los que producen papa comercialmente. Sin embargo los agricultores reconocen que las calidades organolépticas de papas producidas orgánicamente son superiores a aquellas producidas de manera convencional.

A pesar de que la papa no puede ser exportada debido a restricciones fitosanitarias y que varias de la técnicas antes mencionadas se están perdiendo debido a presiones del mercado convencional, existe un mercado alternativo aún incipiente pero con un fuerte crecimiento dispuesto a pagar por el valor agregado de productos 'sanos'. Las técnicas aquí descritas 
pueden contribuir significativamente a desarrollar Sistemas Agrícolas Sostenibles Articulados al Mercado (SAS-M), reduciendo el uso de agro-químicos y economizando la producción de cultivos en zonas alto-andinas. Además en los países encuestados existe el apoyo de instituciones públicas y privadas, empresas certificadoras y ONGs que brindan insumos, asistencia técnica y experiencia en la producción orgánica a gran escala de cultivos de exportación (café, plátano, cacao).

Los retos para el futuro incluyen desarrollar metodologías para diferenciar productos generados en SAS-M, por ejemplo, mediante sellos obtenidos de procesos formales 0 informales de certificación; y desarrollar metodologías para definir el grado de sostenibilidad de dichos sistemas, por ejemplo, mediante el uso de indicadores como el Coeficiente de Impacto Ambiental.

\section{Agradecimientos}

Los autores agradecen a los agricultores que respondieron gentilmente las encuestas en los tres países y compartieron sus percepciones, preocupaciones y conocimientos. A los investigadores y al personal de las ONGs e instituciones públicas y privadas por el apoyo logístico en el contacto con las comunidades. De igual manera, agradecen al proyecto Papa Andina del CIP por el financiamiento del presente estudio.

\section{Literatura Citada}

Agrios, G. N. 1997. Plant pathology. $4^{\text {th }}$. Ed. San Diego, Academic Press.635 p.

Alcázar, J., Cisneros, F. 1999. Taxonomy and bionomics of the Andean potato weevil complex: Premnotrypes spp. and related genera. Impact on a Changing World. Program Report 1997-98. International Potato Center (CIP). Lima Peru. Pp. 141-151.

Altieri, M. 1996. Indigenous knowledge revalued in Andean agriculture. ILEA Newsletter 12(1): 7-11.
Blossiers J., Deza C., León B., Samané, R. 2000. Agricultura de laderas a través de andenes, Perú. En: Manual de Captación y Aprovechamiento del Agua de Lluvia, Experiencias en América Latina, Oficina Regional de la FAO para América Latina y el Caribe, Santiago de Chile, $230 \mathrm{p}$.

Borliaud, J., Herve, D., Morlon, P., Reau, R. 1988. Chakitaklla: Estrategia de barbecho e intensificación de la agricultura andina. ORSTOM - PISA. Lima. 102 p.

Brack, A. 1999. Diccionario enciclopédico de plantas útiles del Perú. Centro de Estudios Regionales Andinos Bartolomé de Las Casas (CBC). Programa de las Naciones Unidas (PNUD). 556 p.

Brush, S., Carney, H., Huamán, Z. 1979. The dynamics of Andean potato agriculture. College of William and Mary. Williamsburg. USA. 23 p.

Canales, N., Canto, R. 2000. Potato moth control with local plants in the storage of potato. En: Natural crop protection in the tropics. Segunda edición. Margraf Verlag. Weikersheim. Alemania: 313-320.

Centro Internacional de la Papa (CIP). 2003. El chuño blanco: Rescatando un sabor ancestral. http://www.cipotato.org/potato/docs/642tunta.pdf. 2 p. Consulta: Junio 2008.

Centro Internacional de la Papa (CIP). 2007. Facts and figures: improvement and conservation. CIP, Lima, Perú. http://www.cipotato.rog/pressroom/facts_figures improvement_conservation.asp. 23 p. Consulta: Junio 2008.

Cóndor, C. 1994. El tipka: un modelo social tecno-agro ecológico andino prehispánico vigente en Aymará - Pazos. Blanco y Negro 1(6): 129-141.

Davis, J. R., Pavek, J. J., Corsini, D. L., Sorensen, L. H., Schneider, A. T., Everson, D. O., Westermann, D. T., and Huisman, O. C. 1994. Influence of continuous cropping of 
several potato clones on the epidemiology of Verticillium wilt of potato. Phytopathology 84: 207-214.

Devaux, A., Velasco, C., López, G., Bernet, T., Ordinola, M., Pico, H., Thiele, G., Horton, D. 2007. Collective Action for Innovation and Small Farmer Market Access: The Papa Andina Experience. CAPRI Working Paper (en edición).

Erickson, C.L. 1984. Waru-waru: una tecnología agrícola del Altiplano Pre-Hispánico. Boletín del Instituto de Estudios Aymaras. 8: 5-37.

Erickson, C.L., Chandler, K.L. 1989. Raised fields and sustainable agriculture in the lake Titicaca basin of Perú. In: JO Browder (ed.). Fragile Lands of Latin America. pp. 230-243. Westview Press, Boulder.

Ewell, P.T., Fuglie, K.O., Raman, K.V. 1994. Farmers perspectives on potato pest management in developing countries: interdisciplinary research at the International Potato Center (CIP). En: G.W. Zehnder (ed.) Advances in potato pest and biology and management. pp: 597-615.

Fonseca, C., Tupac Yupanqui, A., Zuñiga, N., Maldonado, L., Devaux, A. 2002. El almacenamiento de la papa en los andes del Perú y las implicancias técnicas y económicas de una tecnología alternativa. Asociación Latinoamericana de la Papa (ALAP). XX Congreso de la Asociación Latinoamericana de la Papa: Memorias. Lima (Perú). ALAP. 2002. p.132.

Gianella, T. 2004. Chuño blanco, "tunta" o "moraya": un proceso natural de conservación. LEISA. 20, 29-31.

Gomero, L. 2000. Uso de plantas con propiedades repelentes e insecticidas. En: Plantas con potencial biocida. RAAA. Lima. Perú. 13-25 pp.

Gómez, R., Wong, D. 1998. Procesamiento de la papa. Revista Agroecológica Indoagro. Año 1. No. 5. Lima, Perú. 1998
Murcia, G., Barreto, N. 2002. Evaluación de parámetros para el almacenamiento de semilla de papa (Solanum tuberosum) y mecanismos de control de la polilla guatemalteca (Tecia solanivora) durante el mismo. Corporación Colombiana de Investigación Agropecuaria CORPOICA, Colombia. http://www.redepapa.org/murcia.pdf. Consulta: Junio 2008.

Pérez, L. 1996. Crianza de la papa en Paucartambo. CESA. Cusco. Perú. 100 p.

PRATEC. 1987/2002. Tecnologías Campesinas de los Andes. 36 Vol. Lima Perú.

Pumisacho, M., Sherwood, S. 2002. El cultivo de la papa en Ecuador. INIAP-CIP. 229 p.

Tapia, M.E., Fries, A.M. 2007. Guía de campo de los cultivos andinos. Organización de las Naciones Unidas para la Agricultura y la Alimentación (FAO) - Asociación Nacional de Productores Ecológicos del Perú (ANPE-Perú). Lima, Perú. 209 p.

Valdez, L. 1993. El jiri. ProCampo 64: 27-31.

Yabar, E. 1988. Integración de prácticas culturales para el control del gorgojo de los Andes (Premnotrypes spp.). Revista Latinoamericana de la Papa. 1(1): 120-131. 
Anexo 1. Listado de comunidades entrevistadas en Perú, Ecuador y Bolivia.

\begin{tabular}{|c|c|c|c|c|}
\hline País & Provincia & Distrito & Comunidad & $\begin{array}{c}\text { m sobre nivel } \\
\text { del mar } \\
\text { (promedio) }\end{array}$ \\
\hline \multirow{12}{*}{ Perú } & Cajamarca & Namora & Campo Alegre, Huanico, Tallambo Alto & 3700 \\
\hline & San Marcos & Gregorio Pita & Bella Unión & 3500 \\
\hline & Yauyos & $\begin{array}{l}\text { Laraos, San Lorenzo de } \\
\text { Alis, Huantan, Carania }\end{array}$ & $\begin{array}{l}\text { Laraos, San Lorenzo de Alis, Huantan, } \\
\text { Carania }\end{array}$ & $3200-3800$ \\
\hline & Huancayo & Pucará & Patalá & 4100 \\
\hline & Tayacaja & Pazos & $\begin{array}{l}\text { San Cristóbal de Ñahuin, San José de } \\
\text { Aymará }\end{array}$ & 3800 \\
\hline & Angaraes & Lircay, Seclla, Ccochaccasa & $\begin{array}{l}\text { Chahuarma, Buena Vista, Unión } \\
\text { Progreso - Patahuasi, Allato, } \\
\text { Quispicancha, Huaraccopata, Chucuspa, } \\
\text { Millpo, Santa Cruz de Pongos }\end{array}$ & $3200-4100$ \\
\hline & Acobamba & Paucará & $\begin{array}{l}\text { Libertadores Chopcca, Huachhua } \\
\text { Chopcca }\end{array}$ & 3800 \\
\hline & Huancavelica & Yauli & $\begin{array}{l}\text { Dos de Mayo, Sotopampa Chopcca, } \\
\text { Ccasapata }\end{array}$ & $3700-4100$ \\
\hline & Canchis & Checacupe, Sicuani & $\begin{array}{l}\text { Palccoyo, Chapichumo, Acco Acco, } \\
\text { Pataccalasaya, Los Andes }\end{array}$ & 3400 \\
\hline & Chucuito & Pomata & Huapaca Santiago & 3900 \\
\hline & Collao & Ilave & Siale & 3900 \\
\hline & Puno & Capachica & Ocoña & 3900 \\
\hline Ecuador & Chimborazo & Palmira, La Matriz, Cebadas & $\begin{array}{l}\text { Galte Laime, San Pablo de Guantug, } \\
\text { Pancum Ichubamba, Sablog San José, } \\
\text { Gualiñag }\end{array}$ & $2500-3500$ \\
\hline Bolivia & Aroma & & $\begin{array}{l}\text { Catavi, Santari, Huanocollo, Villa } \\
\text { Pucara, Cayaca, Conchamarca, Checa } \\
\text { Belén }\end{array}$ & 4000 \\
\hline
\end{tabular}


Anexo 2. Lista de organizaciones entrevistadas en Perú, Ecuador y Bolivia.

\begin{tabular}{|c|c|c|c|}
\hline $\begin{array}{l}\text { Ámbito } \\
\text { de trabajo }\end{array}$ & Nombre & Dirección y teléfono & Sede \\
\hline Comercialización & Agroimex S.A.C. & $\begin{array}{l}\text { Monterrey } 221 \text { Of. } 215 \text { Surco } \\
\text { 372-0659 }\end{array}$ & $\begin{array}{l}\text { Lima, } \\
\text { Perú }\end{array}$ \\
\hline Comercialización & Comercial Andina Industrial & $\begin{array}{l}\text { Ca. Galeón } 180 \text { Of. } 101 \text { Surco } \\
\text { 271-3366 }\end{array}$ & $\begin{array}{l}\text { Lima, } \\
\text { Perú }\end{array}$ \\
\hline Comercialización & SERFI S.A. & $\begin{array}{l}\text { Av. Benavides } 380 \text { Of. } 401 \\
\text { Miraflores } \\
\text { 446-2896 }\end{array}$ & $\begin{array}{l}\text { Lima, } \\
\text { Perú }\end{array}$ \\
\hline Comercialización & $\begin{array}{l}\text { Corporación Bioquímica } \\
\text { Internacional (CBI) S.A.C. }\end{array}$ & $\begin{array}{l}\text { Av. Argentina } 1959 \text { Callao } \\
\text { 429-6216 }\end{array}$ & $\begin{array}{l}\text { Lima, } \\
\text { Perú }\end{array}$ \\
\hline Comercialización & FARMEX S.A. & $\begin{array}{l}\text { Av. Rivera Navarrete } 620 \\
\text { San Isidro } \\
440-7788\end{array}$ & $\begin{array}{l}\text { Lima, } \\
\text { Perú }\end{array}$ \\
\hline Comercialización & Industrias Químicas S.A.C.- Zijals & $\begin{array}{l}\text { Av. Aviación } 3362 \\
\text { 225-3371 }\end{array}$ & $\begin{array}{l}\text { Lima, } \\
\text { Perú }\end{array}$ \\
\hline Comercialización & Grupo Silvestre & $\begin{array}{l}\text { Ca. Arica } 242 \\
\text { Miraflores } \\
617-3300\end{array}$ & $\begin{array}{l}\text { Lima, } \\
\text { Perú }\end{array}$ \\
\hline Comercialización & $\begin{array}{l}\text { Tecnología Química y Comercio } \\
\text { (TQC) S.A. }\end{array}$ & $\begin{array}{l}\text { Ca. Rene Descartes } 311 \\
\text { Sta. Raquel Ate } \\
\text { 348-1103 }\end{array}$ & $\begin{array}{l}\text { Lima, } \\
\text { Perú }\end{array}$ \\
\hline Comercialización & AGRODEL, Agro Orgánicos & $\begin{array}{l}\text { Luz Elisa Borja } 2940 \text { y Juan } \\
\text { Montalvo, Riobamba } \\
5933296437\end{array}$ & Ecuador \\
\hline Comercialización & BIOSAGRO & $\begin{array}{l}\text { Olmedo } 23 \text { - } 27 \text { y Colón } \\
\text { Riobamba } \\
59332962533\end{array}$ & Ecuador \\
\hline Comercialización & DAVIAGRO & $\begin{array}{l}\text { Av. Bolivariana s/n (frente a la } \\
\text { Villa Ol'impica), Ambato } \\
59332845783\end{array}$ & Ecuador \\
\hline Comercialización & ECUAQUIMICA & $\begin{array}{l}\text { Av. Bolivariana Km } 2 \text { 1⁄2 vía a } \\
\text { Baños, Ambato. } \\
59332840497\end{array}$ & Ecuador \\
\hline Comercialización & $\begin{array}{llll}\text { ESPOCH } & \text { Dpto. } & \text { de } & \text { Ciencias } \\
\text { Biológicas* } & & & \end{array}$ & $\begin{array}{l}\text { Panamericana sur Km 11⁄2, } \\
\text { Riobamba } \\
59332941868\end{array}$ & Ecuador \\
\hline Comercialización & ESPOCH - MIKROBEN & $\begin{array}{l}\text { Panamericana sur Km 11/2, } \\
\text { Riobamba } \\
59332944544\end{array}$ & Ecuador \\
\hline Comercialización & INDIA - PRONACA & $\begin{array}{l}\text { Av. Semillas y Tanasa Km 4 1⁄2 } \\
\text { Vía Durán-Tambo, Guayaquil } \\
59332515533\end{array}$ & Ecuador \\
\hline Comercialización & AGRIPAC & $\begin{array}{l}\text { Juan Montalvo } 14 \text { - } 51 \text { y Boyacá, } \\
\text { Riobamba } \\
59332942219\end{array}$ & Ecuador \\
\hline Comercialización & PROBIOMA & $\begin{array}{l}\text { Equipetrol Calle Cordova Este 29, } \\
\text { Santa Cruz } \\
\text { 591)-33431332 / } 33115031 \text { / } \\
33115032\end{array}$ & Bolivia \\
\hline
\end{tabular}




\begin{tabular}{|c|c|c|c|}
\hline $\begin{array}{l}\text { Ámbito } \\
\text { de trabajo }\end{array}$ & Nombre & Dirección y teléfono & Sede \\
\hline Certificación orgánica & Control Union Perú S.A.C. & $\begin{array}{l}\text { Av. Dos de Mayo } 1205 \text { San Isidro } \\
422-4829\end{array}$ & $\begin{array}{l}\text { Lima, } \\
\text { Perú }\end{array}$ \\
\hline Certificación orgánica & Bio Latina S.A.C. & $\begin{array}{l}\text { Av. Arenales } 645 \text { Jesús María } \\
423-2924\end{array}$ & $\begin{array}{l}\text { Lima, } \\
\text { Perú }\end{array}$ \\
\hline Certificación orgánica & SGS del Perú S.A.C. & $\begin{array}{l}\text { Av. Elmer Faucett } 3348 \text { Callao } \\
517-1900\end{array}$ & $\begin{array}{l}\text { Lima, } \\
\text { Perú }\end{array}$ \\
\hline Certificación orgánica & OCIA & $\begin{array}{l}\text { Ca. Bronsino } 256 \text { San Borja } \\
346-4039\end{array}$ & $\begin{array}{l}\text { Lima, } \\
\text { Perú }\end{array}$ \\
\hline Certificación orgánica & BCS Öko-Garantie & $\begin{array}{l}\text { Av. Camino Real } 348 \text { Torre EL } \\
\text { Pilar Of. 1501-B } \\
7160044\end{array}$ & $\begin{array}{l}\text { Lima, } \\
\text { Perú }\end{array}$ \\
\hline Certificación orgánica & IMO Control Latinoamericana & $\begin{array}{l}\text { Ca. Conquista } 486 \text { Of. } 102 \text { Urb. } \\
\text { J.L.Ortiz Chiclayo } \\
\text { (074)-25-0498 } \\
(074)-25-0518\end{array}$ & $\begin{array}{l}\text { Lima, } \\
\text { Perú }\end{array}$ \\
\hline Certificación orgánica & BCS Öko-Garantie & $\begin{array}{l}\text { Km 31/2 vía a Chamba Riobamba } \\
59332910333\end{array}$ & Ecuador \\
\hline Certificación orgánica & Ecocert Ecuador & $\begin{array}{l}\text { Cevallos Mendiburo } 218 \text { y } \\
\text { Córdova Guayaquil } \\
59342302447\end{array}$ & Ecuador \\
\hline Certificación orgánica & Control Union Perú S.A.C. & $\begin{array}{l}\text { Av. Juan Tanca Marengo y } \\
\text { Constitución, 4to piso Edificio } \\
\text { Novis Guayaquil } \\
59342224829\end{array}$ & Ecuador \\
\hline Certificación orgánica & $\begin{array}{l}\text { Certification of Environmental } \\
\text { Standards GmbH - CERES }\end{array}$ & $\begin{array}{l}\text { Acacia } 1011 \text { y Urseda Central } \\
\text { Guayaquil }\end{array}$ & Ecuador \\
\hline Certificación orgánica & $\begin{array}{l}\text { AOPEB Asociación de Organizaciones } \\
\text { de Productores Ecológicos de Bolivia }\end{array}$ & $\begin{array}{l}\text { Av. Landaeta 554, La Paz } \\
\text { 591-2-2490686 / } 2490691 \text { / } \\
2129287\end{array}$ & Bolivia \\
\hline
\end{tabular}

